\title{
Important Notice
}

The article entitled ' Gary, Indiana: Steeltown Extraordinary' which appeared in Volume 9, Part I, drew heavily on an undergraduate dissertation written, under Mr Brook's supervision, by Mr Michael Turner, now of the State University of New York at Binghamton. All parties concerned (Mr Turner, Mr Brook, the Editor and the publishers) agree that it would be proper for $\mathrm{Mr}$ Turner's name to be given as co-author in any citation. Accordingly the article is being jointly indexed in the Journal.

We would like, at the same time, to apologise for and to correct the accidental omission of one line of type in the article as printed. On page $3^{8}$ the final sentence of the penultimate paragraph in section I should read: In 1905 the total population of the Calumet region was only 19,000: Gary did not exist, Whiting and East Chicago were little more than villages, and Hammond, the oldest of the cities of the Calumet, had only 12,000 inhabitants. 